\title{
Step-edge sputtering through grazing incidence ions investigated by scanning tunneling microscopy and molecular dynamics simulations
}

\author{
Alex Redinger, ${ }^{1,2, *}$ Yudi Rosandi, ${ }^{3}$ Herbert M. Urbassek, ${ }^{3}$ and Thomas Michely ${ }^{1}$ \\ ${ }^{1}$ II. Physikalisches Institut, Universität zu Köln, 50937 Köln, Germany \\ ${ }^{2}$ I. Physikalisches Institut, RWTH-Aachen, 52056 Aachen, Germany \\ ${ }^{3}$ Fachbereich Physik, Technische Universität Kaiserslautern, 67663 Kaiserslautern, Germany
}

(Received 15 February 2008; published 23 May 2008)

\begin{abstract}
Scanning tunneling microscopy is used to quantify step-edge sputtering of $\mathrm{Pt}(111)$ at $550 \mathrm{~K}$ by grazing incidence ion bombardment with $5 \mathrm{keV} \mathrm{Ar}^{+}$ions. For bombardment conditions causing negligible erosion on terraces, damage features associated with step bombardment allow us to visualize step retraction and thus to quantify the step-edge sputtering yield. An alternative method for step-edge yield determination, which is applicable under more general conditions, is the analysis of the concentration of ascending steps together with the removed amount as a function of ion fluence. Interestingly, the azimuthal direction of the impinging ions with respect to the surface significantly changes the sputtering yield at step edges. This change is attributed to the orientation dependence of subsurface channeling. Atomistic insight into step-edge sputtering and its azimuthal dependence is given by molecular dynamics simulations of ion impacts at 0 and $550 \mathrm{~K}$. The simulations also demonstrate a strong dependence of the step-edge sputtering yield on temperature.
\end{abstract}

DOI: 10.1103/PhysRevB.77.195436

\section{INTRODUCTION}

Patterning of metallic and semiconductor surfaces through grazing incidence ion bombardment has gained increasing interest in the last decade. The ability to create nanogrooves and ripple patterns ${ }^{1-6}$ with tunable lateral periodicity and amplitude has several potential applications. The resulting morphology can be used as a template for the adsorption of large molecules ${ }^{7}$ for the manipulation of magnetism ${ }^{8}$ or for tuning the chemical reactivity of catalytically active surfaces. ${ }^{9}$ Grazing incidence ion beams are also of interest in ion beam assisted deposition ${ }^{10}$ and were shown to effectively change texture and roughness of thin films. ${ }^{11}$

A practical prerequisite for future applications of grazing incidence ion beams is knowledge of how fast the surface erodes for a given ion flux of a specific energy. For normal incidence ion bombardment, the erosion rate is largely temperature and morphology independent. The sputtering yield $\bar{Y}$ - the average number of sputtered particles per incident ion - may be readily calculated with fair accuracy by analytical formulas ${ }^{12,13}$ or Monte Carlo simulations based on the binary collision approximation [e.g., TRIM (Ref. 14)]. Knowledge of this is thus sufficient for the prediction of erosion rate.

For grazing incidence ions, the situation is considerably more difficult. The erosion rate or the average sputtering yield $\bar{Y}$ is a sensitive function of the surface morphology and, thus, also of temperature and ion fluence, as we have shown in our previous work. ${ }^{15}$ Whereas the ion is reflected from flat terraces without sputtering-this process is termed surface channeling in the ion-surface scattering community-large angle scattering and significant sputtering take place if ions interact with step atoms or point defects.

In this paper, we describe two methods of measuring the sputtering yield $Y^{\text {step }}$ characterizing the interaction of grazing incidence ions with surface steps. The first, which is a very direct method, visualizes $Y^{\text {step }}$ by measuring step retraction through scanning tunneling microscopy (STM) topographs.
PACS number(s): 79.20.Rf, 68.49.Sf, 31.15.xv, 68.37.Ef

While this method is only applicable under extremely grazing incidence conditions, the second method is generally more applicable. It relies on the determination of the step concentration and on measuring the removed material. We show that with knowledge of the step-edge concentration and of the normal incidence amorphous yield $Y^{\text {amorph }}$, the fluence dependent global erosion rate or $\bar{Y}$ may be predicted with an accuracy of a factor of 2. Finally, with the help of moleculardynamics (MD) simulations, we are able to understand the significant dependence of $Y^{\text {step }}$ on the azimuthal direction and find a strong dependence of the yield on temperature.

\section{GEOMETRICAL MODEI}

Above, we already referred to the step-edge yield $Y^{\text {step }}$. To clarify terminology and as a prerequisite for the presentation of the experimental results, we briefly summarize some of our previous work related to sputtering and damage at grazing incidence ion bombardment. ${ }^{15,16}$ As sketched in Fig. 1(a), for the grazing incidence geometry, we distinguish two classes of ion trajectories. The first class consists of ions hitting an ascending (illuminated) step edge either indirectly, after reflection from the lower terrace [trajectory labeled 1 in Fig. 1(a)], or directly [trajectory labeled 2 in Fig. 1(a)]. The second class consists of all other ions from which the majority consists of ions hitting the terrace [trajectory labeled 3 in Fig. 1(a)]. Also, ions approaching the surface at or close to descending steps belong to this class. We label the distance of the ion penetration point through the plane of the upper terrace layer nuclei [dashed line in Fig. 1(a)] to the step-edge atom nucleus by $\xi$. All ions with $\xi \in\left[-x_{c}, 0\right]$ belong to the first class. Here, $x_{c}$ characterizes the width of the zone of influence with $\Delta h$ being the step height; it is $x_{c}=2 \Delta h \tan \vartheta$ from geometry.

The ions of the first class enter the zone of influence in front of an illuminated step edge [compare Fig. 1(b)]. Thus, they hit the step edge and cause step-edge sputtering with a yield $Y^{\text {step }}$. For all other ions, a terrace impact takes place 
(a)
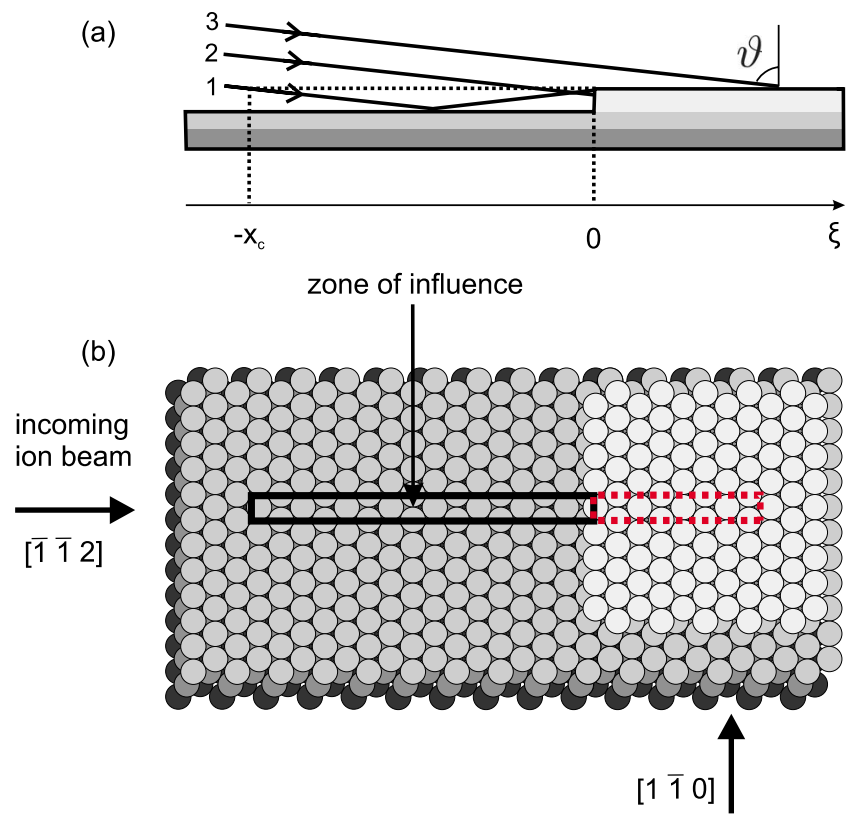

FIG. 1. (Color online) (a) Schematic sketch of the geometrical model (see text). (b) Ball model of an fcc(111) surface displaying the two azimuthal directions of ion bombardment used by the arrows. Along the [ $\overline{1} \overline{1} 2]$ azimuth, the ions impinge on dense packed $\{111\}$-faceted steps, while along the [1피 azimuth, the ions impinge on open fully kinked steps. For the [112] azimuth and $\vartheta$ $=83^{\circ}$, a one atom wide strip of influence is indicated as a solid box. Atoms crossing the area of the solid box at the level of the upper terrace will schematically remove a strip of atoms indicated by the dotted box [here, 8.4 atoms (Ref. 15)].

with a low sputtering yield $Y^{\text {terr }}$. If $A^{\text {step }}$ is the fractional area of the zones of influence of the ascending steps, $1-A^{\text {step }}$ is the fractional area of the remaining surface. With these definitions, the average sputtering yield $\bar{Y}$ is simply

$$
\bar{Y}=A^{\text {step }} Y^{\text {step }}+\left(1-A^{\text {step }}\right) Y^{\text {terr }} .
$$

Note that $\bar{Y}$ is identical to the average erosion rate,

$$
\bar{Y}=\frac{d \Theta}{d F},
$$

if the eroded amount $\Theta$ and the ion fluence $F$ are measured in monolayer (ML) and monolayer equivalent (MLE), respectively. By using this model for $5 \mathrm{keV} \mathrm{Ar}^{+}$impinging on $\mathrm{Pt}(111)$ with a polar angle of $83^{\circ}$ along the [11 2$]$ azimuth, we experimentally obtained $Y^{\text {step }}=8.4 \pm 1.5$ and $Y^{\text {terr }}=0.08$ [compare also Fig. 1(b)]. ${ }^{15}$ MD simulations corroborated that indeed for these parameters, the sputtering yield is negligible or zero everywhere except within a zone along the step edge coinciding with an accuracy of one atomic row spacing with the geometrical zone of influence. ${ }^{15,16}$

The application of the model is meaningful if the sputtering from the terraces is small. Such conditions are met within or close to the surface channeling regime. For surface channeling, the normal component of the kinetic energy $E_{\perp}$ $=E \cos ^{2} \vartheta$ is below the critical energy $E_{\text {crit }}$, above which surface channeling ceases (where $E$ denotes the total ion ki- netic energy). ${ }^{17}$ In the channeling regime, $E_{\perp}$ is too low to overcome the repulsive collective potentials of surface atom strings or surface atom planes. Consequently, large angle scattering events do not take place and sputtering is absent. ${ }^{18}$

Due to its simplicity, the geometrical model allows us to describe the erosive interaction of grazing incidence ions with surfaces by just two numbers, of which $Y^{\text {terr }}$ is of the order of zero and $Y^{\text {step }} \approx Y^{\text {amorph }}$. The fact that two different methods of step-edge yield determination have identical results for $Y^{\text {step }}$ (as shown below) lends additional credit to this concept and makes it a useful tool for the estimation of erosion rates.

According to the geometrical model, one would expect that (i) the sputtering yield does not depend on the value of $\xi$ as long as $\xi \in\left[-x_{c}, 0\right]$, (ii) the step-edge sputtering yield does not depend on the azimuthal angle, (iii) $Y^{\text {step }}$ is largely temperature independent, and (iv) the step-edge sputtering yield is largely independent of the angle of incidence as long as the terrace damage is negligible. As we will see below, all four expectations fail. The common origin of these failures is the neglect of planar and axial subsurface channeling. Grazing incidence ions may enter at ascending steps into the crystal and may be guided over long distances away from the step, thereby obtaining less violent pathways for energy loss (e.g., electronic) and resulting in less sputtering. While we have previously shown ${ }^{4,5}$ that subsurface channeling is of crucial importance for pattern formation, we will see below that it is also responsible for significant variations of $Y^{\text {step }}$ with the polar and azimuthal angle within the zone of influence and with temperature.

\section{EXPERIMENTAL AND COMPUTATIONAL METHODS}

The experiments were performed in an ultrahigh vacuum variable temperature STM apparatus with a base pressure in the $10^{-11}$ mbar range. ${ }^{19}$ Sample cleaning was accomplished by cycles of ion bombardment and flash annealing at $1273 \mathrm{~K}$. A mass separated $5 \mathrm{keV} \mathrm{Ar}^{+}$ion beam is used to bombard $\mathrm{Pt}(111)$ along the $[\overline{1} \overline{1} 2]$ or the $[1 \overline{1} 0]$ direction [see Fig. 1(b)]. The ion flux $f$ is measured with a Faraday cup prior to each experiment and is set between $1.0 \times 10^{16}$ ions $\mathrm{m}^{-2} \mathrm{~s}^{-1}(\vartheta$ $\left.=86^{\circ}\right)$ and $2.0 \times 10^{16}$ ions $\mathrm{m}^{-2} \mathrm{~s}^{-1}\left(\vartheta=82^{\circ}-83^{\circ}\right)$. The ion fluence $F=f t$ is measured below in MLEs, where $1 \mathrm{MLE}$ equals the areal density of surface layer atoms on $\mathrm{Pt}(111)$ $\left(1.504 \times 10^{19}\right.$ atoms $\left.\mathrm{m}^{-2}\right)$. For the two azimuthal orientations investigated, the sample is remounted in the sample holder. The sample orientation is controlled by analyzing the angle between $\langle 110\rangle$ oriented steps resulting from slip induced by tip crashes and a well developed ripple pattern fabricated according to the recipes of Ref. 4 . The accuracy of azimuthal sample orientation is about $\pm 2^{\circ}$. The adjustment of the polar angle of ion incidence is described in Ref. 4.

In the experiments, the sample temperature during ion exposure was set to $550 \mathrm{~K}$, allowing straightforward data interpretation. The vacancies produced during the bombardment have limited mobility and agglomerate to vacancy islands at the surface, thereby directly visualizing the locations of erosion. The target adatoms produced due to the ion exposure are mobile and heal rather completely. ${ }^{20}$ Step-edge diffusion is efficient at $550 \mathrm{~K}$, keeping the visible structures 

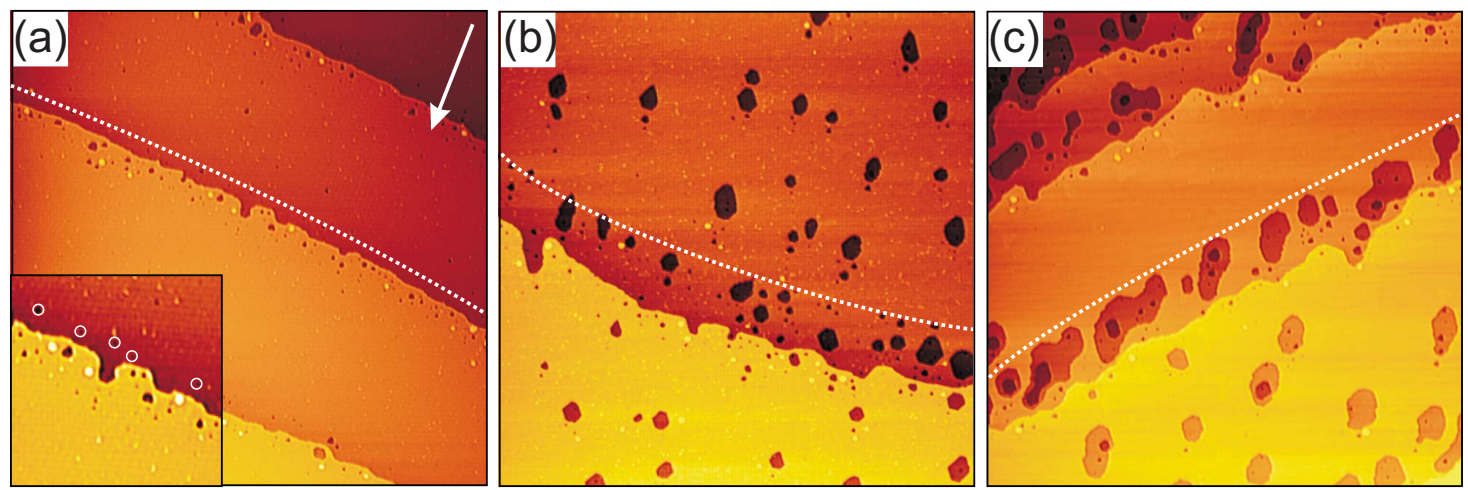

FIG. 2. (Color online) STM topographs after exposure to (a) $0.25 \mathrm{MLE}$, (b) $1.0 \mathrm{MLE}$, and (c) $1.5 \mathrm{MLE} 5 \mathrm{keV} \mathrm{Ar}^{+}$at $550 \mathrm{~K}$ along the [1112] azimuth with $\vartheta=86^{\circ}$. The direction of the ion beam is indicated by a white arrow in (a). The ion flux was set to 1.0 $\times 10^{16} \mathrm{ions} /\left(\mathrm{m}^{2} \mathrm{~s}\right)$. All steps visible are of monolayer height. The inset in (a) shows vacancy clusters (circled) in front of an ascending step edge. The image size is $2450 \AA \times 2450 \AA$, and the size of inset is $700 \times 700 \AA^{2}$.

compact. ${ }^{5}$ Only the diffusion of vacancies created below the surface is still hampered, making annealing experiments necessary for the determination of their extent. ${ }^{21}$

The MD simulation procedure for the calculation of the $83^{\circ}$ data is briefly described in the following. ${ }^{15,16}$ We use a $\mathrm{Pt}(111)$ crystallite, which consists of roughly 50000 atoms, arranged in 17 layers; the lateral dimensions amount to about $200 \times 90 \AA^{2}$ for both azimuths. Its (111) surface is free and relaxed; the other five sides contain three layers of damped atoms in order to simulate the environment of the crystallite; the outermost layers are fixed. ${ }^{22}$ We employ a many-body interaction potential ${ }^{23}$ to describe the Pt interatomic interaction, which has been splined at high energies to the $\mathrm{ZBL}^{24}$ potential. The Ar-Pt interaction is modeled to be purely repulsive according to the ZBL potential. We simulated the processes occurring after ion impact for at least $10 \mathrm{ps}$. The simulation data shown here refer to a target temperature of 0 $\mathrm{K}$, unless otherwise indicated. A number of simulations have also been performed at $550 \mathrm{~K}$, which is the temperature of ion exposure in the experiments. We use velocity scaling to reach this temperature in the target and let it relax for at least 25 ps to reach equilibrium. We note that for these simulations, it was essential to fix the bottommost layer of the crystallite in order to stabilize it against "floppy" longwavelength vibrational and torsional modes.

We also performed MD simulations for other incidence angles. Especially for the $\vartheta=86^{\circ}$ data, the lower terrace of the simulation crystallite had to be enlarged in the direction of the incident ion; the simulation crystallite extends to 260-280 $\AA$ compared to $200 \AA$ for $83^{\circ}$ incidence angle. For this case, the crystallite has 62 000-66 000 atoms. Due to the strongly increased simulation times, only the $0 \mathrm{~K}$ case was simulated.

\section{RESULTS}

\section{A. Step retraction due to ion bombardment}

Figure 2 shows a series of experiments with the ion beam along the [1-12] azimuth after ion exposure at extremely grazing incidence with $\vartheta=86^{\circ}$. All STM images show one or more preexisting steps. The direction of the ion beam is in- dicated by a white arrow in Fig. 2(a). For the lowest fluence in Fig. 2(a), essentially no damage is present on the terraces. As highlighted by the inset, however, behind the exposed preexisting steps, small vacancy islands and a few adatom islands are visible. Close inspection shows that also in front of the preexisting steps, small vacancy islands are apparent [circled in Fig. 2(a)]. As we will argue below, these little vacancy islands mark the initial position of the preexisting step edges [white dashed lines in Fig. 2(a)]. Increasing the ion fluence [compare Figs. 2(b) and 2(c)] causes a pronounced growth in number and size of the vacancy islands in front of the preexisting steps. In addition, large vacancy islands with low number density are now also present on terraces.

The appearance of vacancy islands on the terraces in Figs. 2(b) and 2(c) may be understood as follows. For $\vartheta=86^{\circ}$ with $E^{\perp}=24 \mathrm{eV}$, sputtering from clean terraces is zero (compare also Sec. IV C.). However, due to thermal vibrations, adatom-vacancy pairs may be produced. The liberated adatoms - also occasionally adsorbed molecules from the background gas-may cause a few sputtering events to occur, ${ }^{25}$ resulting in an excess of surface vacancies. After a considerable induction time, these mobile surface vacancies may be sufficient in number to aggregate to form small vacancy clusters. As these vacancy clusters expose themselves as an ascending step to the ion beam, once formed, they rapidly grow to the visible size through step-edge impacts.

The presence of vacancy clusters behind the step edges is apparent in all three topographs of Fig. 2 results from subsurface channeling. ${ }^{5}$ Ions hitting the ascending steps at the bottom of the step enter into the crystal with high probability and are guided in between the planar potentials of the first and the second layer. The ions oscillate between the two layers and will dechannel after they moved a distance dependent on surface temperature. At the location of dechanneling, adatom and vacancy clusters are created, which are large enough to be grown with significant probability by subsequent step-edge impacts to visible vacancy islands.

Important for the following is the appearance of vacancy islands in front of the step edge, which grow in number and size with ion fluence. The dashed lines in all topographs of Fig. 2 indicate the positions where the density of vacancy 


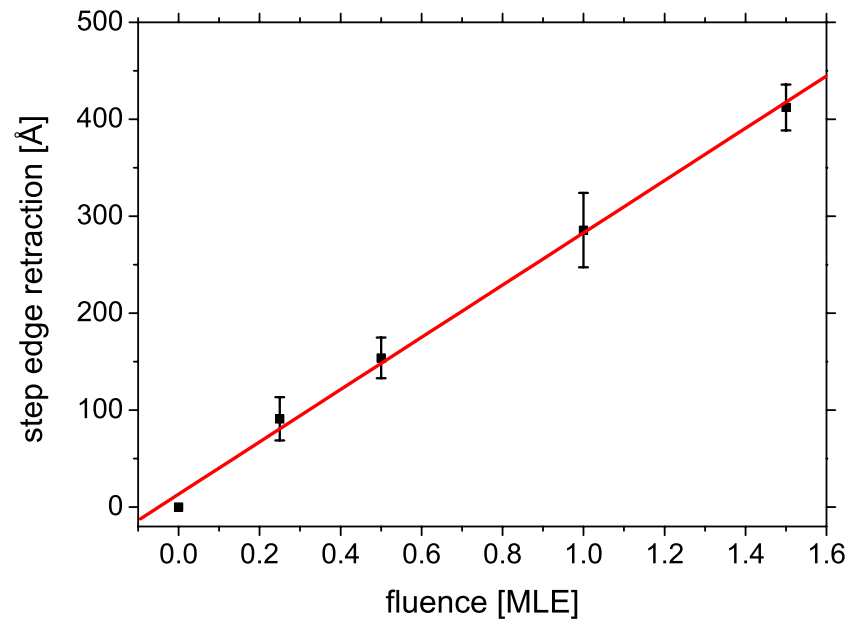

FIG. 3. (Color online) Step retraction $x_{\text {step }}$ as a function of the ion fluence. A linear fit to the data yields a step retraction of $270 \AA \pm 9 \AA /$ MLE.

clusters starts to increase in comparison to the flat terrace. These dashed lines mark the initial positions of the preexisting steps according to the following arguments. Step-edge impacts cause thermal spikes and result in large numbers of adatoms and vacancies. Molecular dynamics simulations show that vacancies are created not only in the upper terrace but also in the lower terrace and, to a certain extent, in the subsurface layers. ${ }^{4,16}$ Due to the step-edge barrier for vacancies, the lower terrace vacancies do not anneal at the nearby ascending step edge $\mathrm{e}^{26,27}$ and thus form with significant probability vacancy islands. These islands grow through subsequent step-edge impacts in the direction of the ion beam to the large structures visible in Figs. 2(b) and 2(c). Through diffusion, they also efficiently collect the lower terrace vacancies originating from step-edge impacts into the preexisting steps at other locations.

As the positions of the dashed lines in Fig. 2 mark the initial positions of the step edges, measuring their average distances from the preexisting steps in the ion beam direction allows us to obtain the step retraction $x_{\text {step }}$ as a function of fluence $F{ }^{28} x_{\text {step }}$ is a linear function of $F$ resulting in a step velocity $v_{\text {step }}=x_{\text {step }} / F=270 \pm 9 \AA \mathrm{MLE}^{-1}$ for the $[\overline{1} \overline{1} 2]$ azimuth (compare Fig. 3) and 216 $\pm 45 \AA \mathrm{MLE}^{-1}$ for the [1 $\left.\overline{1} 0\right]$ azimuth (data not shown). The geometrical model links $x_{\text {step }}$ with $Y^{\text {step }}$ through

$$
x_{\text {step }}=\frac{x_{c}}{\Delta x} F Y^{\text {step }} \Delta x,
$$

where $\Delta x$ is the distance between two atoms on the surface parallel to the ion beam direction $(\Delta x=2.40 \AA$ in the [1 12$]$ direction and $\Delta x=2.78 \AA$ in the [110] direction). Equation (3) attests that the step retraction $x_{\text {step }}$ is the number of ions entering the zone of influence $x_{c} F / \Delta x$ times the retraction of the step edge in ion beam direction $Y^{\text {step }} \Delta x$ caused by each of these ions. Rewriting Eq. (3) leads to

$$
Y^{\text {step }}=\frac{x_{\text {step }}}{x_{c} F}
$$

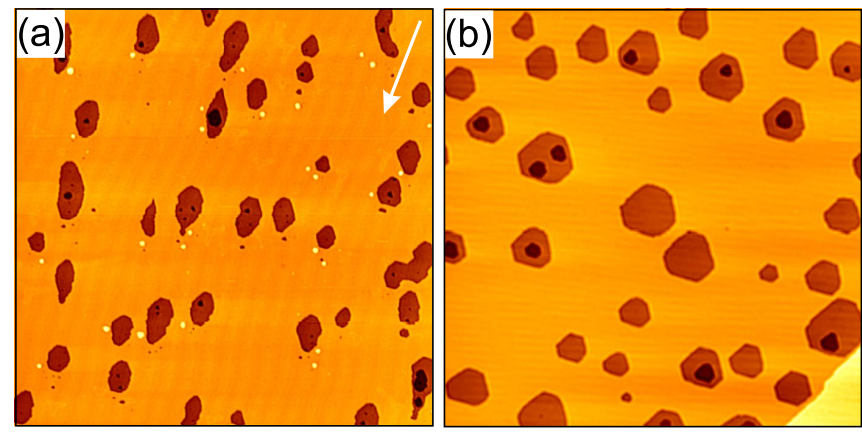

FIG. 4. (Color online) (a) STM topograph of Pt(111) after exposure to $1.5 \mathrm{MLE}$ of $5 \mathrm{keV} \mathrm{Ar}^{+}$at $550 \mathrm{~K}$ along the [1]12] azimuth with $\vartheta=86^{\circ}$. (b) After additional annealing for $120 \mathrm{~s}$ at $720 \mathrm{~K}$. The direction of the ion beam is indicated by a white arrow in (a). The image size is $2450 \times 2450 \AA^{2}$.

The application of Eq. (4) results in $Y^{\text {step }}=4.2$ and $Y^{\text {step }}$ $=3.3$ for the $[\overline{1} \overline{1} 2]$ azimuth and $[1 \overline{1} 0]$ azimuth, respectively. However, these numbers are not yet the proper values for $Y^{\text {step }}$ as they do not take into account that at $550 \mathrm{~K}$ the subsurface vacancies created during step retraction do not completely anneal at the surface and are thus not counted. ${ }^{29}$ Figure 4 compares STM topographs after exposure to 1.5 MLE $5 \mathrm{keV} \mathrm{Ar}^{+}$at $550 \mathrm{~K}$ [Fig. 4(a)] and with additional annealing for $120 \mathrm{~s}$ at $720 \mathrm{~K}$ [Fig. 4(b)]. Annealing at $720 \mathrm{~K}$ is sufficient to remove all subsurface vacancies and to make them visible as surface vacancies. ${ }^{29}$ Due to annealing, the apparent removed amount increases by 35\%. Taking this into account, $Y^{\text {step }}$ is $5.7 \pm 0.2$ along the [1 12$]$ azimuth and $4.5 \pm 0.9$ along the $[1 \overline{1} 0]$ azimuth. Finally, we note that in Figs. 2(b), 2(c), and 4(a), usually large vacancy islands on the terrace are accompanied by small daughter vacancy islands situated behind the large one with respect to the ion beam direction. These daughter vacancy islands originate from the dechanneling damage caused by ions hitting the illuminated steps of the large mother vacancy islands. ${ }^{4,5}$

\section{B. Step-edge yield from step concentration}

Figure 5 shows two series of topographs after exposure to increasing ion fluences. The left column [Figs. 5(a)-5(c)] displays the effect of ion bombardment along the [1̄12] azimuth with $\vartheta=83^{\circ}$ and the right one along the [1 $\left.1 \overline{1} 0\right]$ azimuth with $\vartheta=82^{\circ}$. In comparison to Fig. 2, the number of vacancy islands on the terraces in Fig. 5 is dramatically higher for similar ion fluences. This increase is due to the lowering of $\vartheta$ by a few degrees. It results in an enhanced production of mobile vacancies on the terraces $\left(Y^{\text {terr }} \neq 0\right)$, which aggregate to small vacancy islands and subsequently grow by step-edge impacts. ${ }^{30}$ Under such conditions, $Y^{\text {step }}$ can no longer be obtained through monitoring of step retraction.

The three rows of Fig. 5 represent the three stages of pattern formation onset: (i) aligned formation of vacancy islands through subsurface channeling, (ii) preferential coalescence of the vacancy islands along the ion beam direction as a consequence of their alignment, and (iii) onset of 

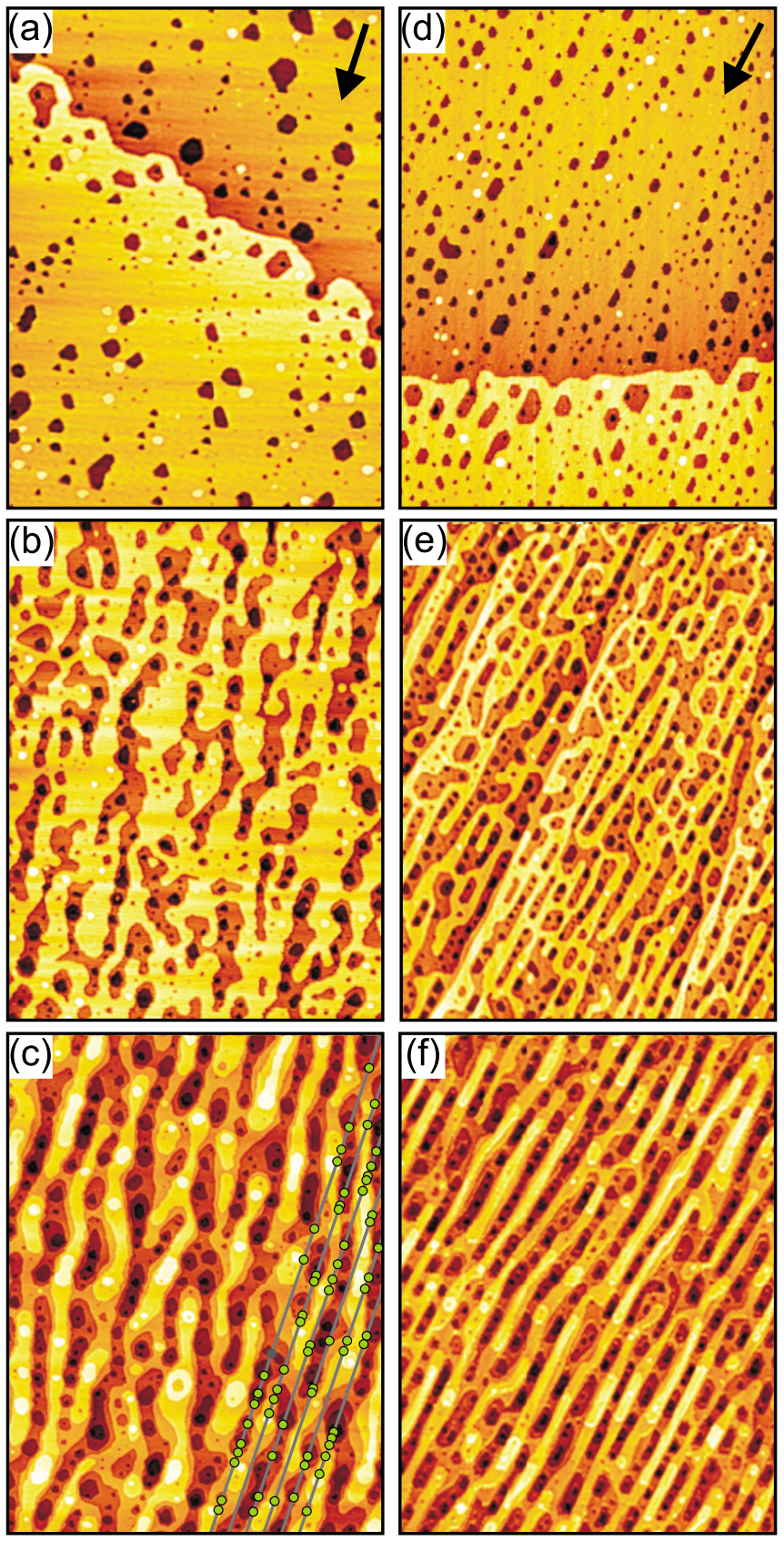

FIG. 5. (Color online) STM topographs of Pt(111) after exposure to (a) 0.5 MLE, (b) 1.0 MLE, (c) 2.0 MLE, (d) 0.57 MLE, (e) 1.1 MLE, and (f) $2.3 \mathrm{MLE}$ of $5 \mathrm{keV} \mathrm{Ar}^{+}$at $550 \mathrm{~K}$. For (a)-(c), the ion bombardment is along the [11 2$]$ azimuth with $\vartheta=83^{\circ}$ and for (d)-(f), along the [1 $1 \overline{1} 0]$ azimuth with $\vartheta=82^{\circ}$. The direction of the ion beam is indicated by a black arrow in (a) and (d). The image sizes are $1200 \times 1600 \AA^{2}$ in (a) and (d) and $1840 \times 2450 \AA^{2}$ in (b), (c), (e), and (f).

multilayer groove formation through the step-edge barrier for vacancies. For a detailed description of these morphological stages, the reader is referred to Refs. 4 and 5.

However, a striking difference in the pattern evolution for the two azimuthal orientations has to be mentioned here. An aligned formation of vacancy islands (chains of vacancy islands) is much more pronounced in the [1 $1 \overline{10}]$ azimuth [Fig.

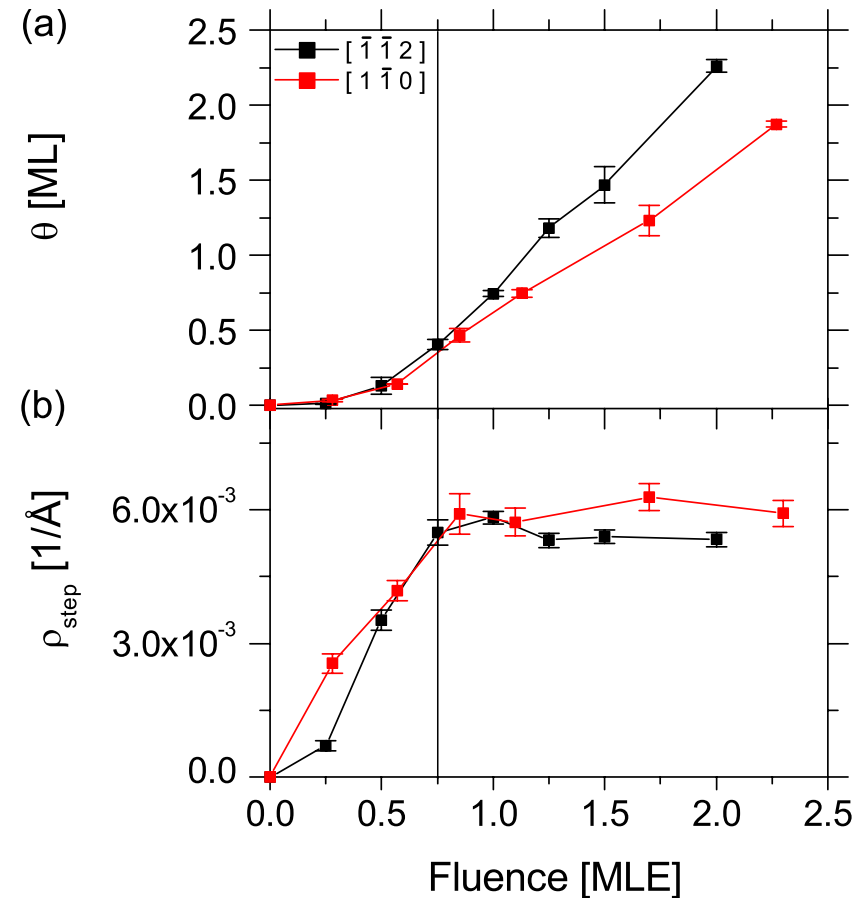

FIG. 6. (Color online) (a) Removed material $\Theta$ versus fluence $F$ for bombardment conditions as in Fig. 5. (b) Concentration of ascending steps $\rho_{\text {step }}$ parallel to the ion beam direction versus $F$. The

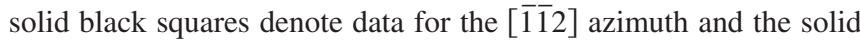
red circles denote data for the $[1 \overline{1} 0]$ azimuth.

5(d)] compared to the [ $\overline{1} \overline{1} 2]$ azimuth [Fig. 5(a)]. This difference causes, in the subsequent stages of pattern formation, a better alignment of the pattern to the ion beam direction and a high pattern regularity for bombardment along the [1 $\overline{1} 0]$ azimuth. This better alignment can be traced back to the fact that axial subsurface channeling takes place along the $[1 \overline{1} 0]$ direction, leading to a better aligned and more distant damage production behind a vacancy island, which thus initiates a better aligned growth of a daughter vacancy island. In contrast, in the [112] direction, planar subsurface channeling takes place, which is less efficient and where the ion trajectories are much easier deflected from their initial direction through thermal vibrations (compare also the discussion in Sec. IV C).

For the two sequences of experiments, which are partly visualized in Fig. 5, the increase in the removed amounts of $\Theta$ with $F$ was measured in incremental steps through grayscale discrimination of STM topographs. Figure 6(a) displays the change of $\Theta$ with $F$ for the two different azimuths. After an initial slow onset of erosion, $\Theta$ linearly increases with ion fluence for $F \geq 0.75$ MLE. According to Eq. (2), the erosion rate $\bar{Y}$ is the slope of change of $\Theta$ with $F$. Thus, for both azimuths, $\bar{Y}$ is initially small and then saturates to $\bar{Y}$ $=1.5$ for the $[\overline{1} \overline{1} 2]$ azimuth and to $\bar{Y}=1.0$ for the $[1 \overline{1} 0]$ azimuth. The values given are the results of linear fits for $F$ $\geq 0.75$ MLE.

The concentration of ascending steps was analyzed for the two sequences of experiments in the following way. As 
shown in Fig. 5(c), lines parallel to the ion beam direction, which are in a distance below and incommensurate with the ripple pattern wavelength, are overlayed to each topograph. Counting the number of ascending steps divided by the total line length along the projection of the ion beam direction onto the surface results in a density of ascending steps $\rho_{\text {step }}$. In Fig. 6(b), $\rho_{\text {step }}$ is plotted versus $F$. It rapidly increases with $F$ and saturates for $F \geq 0.75$ MLE at $\rho_{\text {step }} \approx 6 \cdot 10^{-3} 1 / \AA$.

From the comparison of Fig. 6(b) to Fig. 6(a), it is apparent that the increase in the step-edge concentration is mirrored in the increase in the slope of $\Theta$ versus $F$, i.e., of $\bar{Y}$. The simultaneous saturation of $\rho_{\text {step }}$ and the slope of $\Theta$ versus $F$ directly visualizes that $\bar{Y}$ is determined by $\rho_{\text {step. }}$. By using the linear fits for $\bar{Y}$ and $\rho_{\text {step }}$ for $\Theta \geq 0.75$ MLE and with the application of

$$
Y^{\text {step }}=\frac{\bar{Y}}{\rho_{\text {step }} x_{c}},
$$

we obtain $Y^{\text {step }}=7.4 \pm 0.3$ for the [11 2$]$ azimuth and $Y^{\text {step }}$ $=4.5 \pm 0.2$ for the $[1 \overline{1} 0]$ azimuth.

An error discussion is in place here. With the application of Eq. (5), we neglected terrace sputtering. As the terrace yield is small, it only insignificantly contributes to sputtering. Here, $Y^{\text {terr }}=0.08$ and as $A^{\text {step }} \approx 25 \%$, the contribution of terrace sputtering to $\bar{Y}$ is roughly $5 \%$, causing a slight overestimation of $Y^{\text {step }}$. On the other hand, all ascending steps are counted for the determination of $\rho_{\text {step }}$, although due to multiple steps or shadowing by ascending steps for some of them, the zone of influence is smaller than $x_{c}$. This causes an underestimation of $Y^{\text {step }}$. A detailed analysis of the step distribution shows that roughly $8 \%$ of the ascending step edges have a smaller zone of influence than that of the geometrical model. Since both errors counteract each other, the overall underestimation of the sputtering yield is $3 \%$.

Finally, for establishing $Y^{\text {step }}$, we neglected, for a good reason, the low fluence data below $\Theta \leq 0.75$ MLE. As pointed already out above, step-edge impacts also cause production of subsurface vacancies invisible at the surface. However, after a certain ion fluence, their concentration saturates due to a dynamic equilibrium between damage production and annealing of subsurface damage to the surface. Previous normal incidence experiments indicate that the subsurface damage saturates for ion fluences well below 0.75 MLE. ${ }^{31,32}$ Therefore, for $\Theta \geq 0.75$ MLE, the erosion rate is fully represented by changes at the surface. Neglecting the low fluence data thus makes additional annealing avoidable. By taking into account all errors described above, the stepedge sputtering yields are parallel to the [11 2$]$ azimuth equal to $7.7 \pm 0.9$ and along the $[1 \overline{1} 0]$ azimuth equal to $4.6 \pm 0.6$.

\section{Molecular dynamics simulations}

In the first set of MD simulations, we investigated the dependence of the terrace yield for sputtering $Y^{\text {terr }}$ and of the terrace yield for adatom production $Y^{\text {terr,ad }}$ on the incidence angle $\vartheta$. The simulations were performed for the [112] azimuth and each data point represented in Fig. 7 averages over

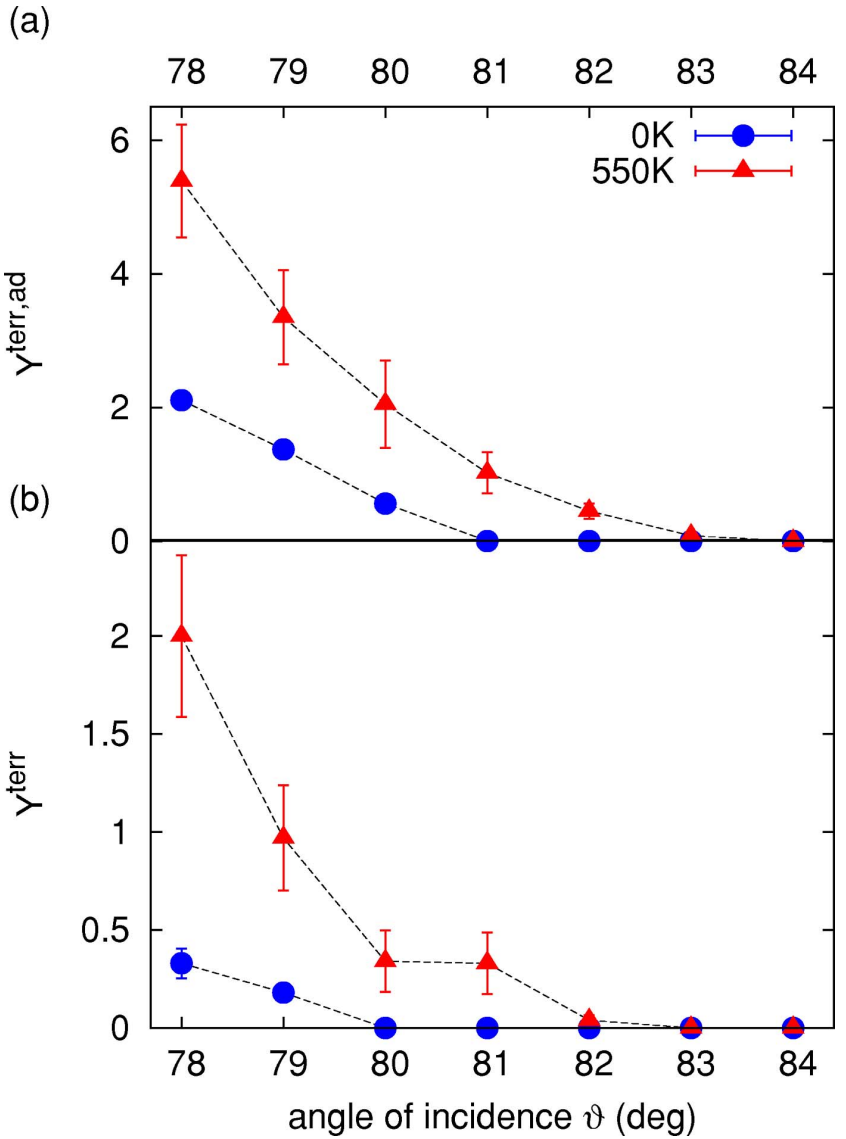

FIG. 7. (Color online) (a) Terrace adatom yield $Y^{\text {terr,ad }}$ and (b) terrace sputter yield $Y^{\text {terr }}$ as a function of the polar angle $\vartheta$ for 5 $\mathrm{keV} \mathrm{Ar}^{+}$incident on $\mathrm{Pt}(111)$ along the [1]12] azimuth. Circles: MD simulations at $0 \mathrm{~K}$; and triangles: $\mathrm{MD}$ simulations at $550 \mathrm{~K}$.

100 ion impacts. Figure 7 visualizes the strong decrease in these yields as the polar angle approaches $90^{\circ}$. Independently of the temperature of simulation, $Y^{\text {terr }}$ ceases for smaller $\vartheta$ than $Y^{\text {terr,ad }}$. This result reflects the fact that less kinetic energy needs to be transferred for the production of an adatom still bound to the surface compared to the sputtering of an atom. Compared to the $0 \mathrm{~K}$ simulations, the $550 \mathrm{~K}$ yields diminish only for larger $\vartheta$. For the arriving ions, the significant vibration amplitude of surface atoms at $550 \mathrm{~K}$ causes deviations from the planar surface potential and the probability for large angle scattering events increases.

Figure 8 displays $Y^{\text {terr }}$ at $0 \mathrm{~K}$ for the [112] azimuth over a wider range of polar angles $\vartheta$. The curve connecting the data resembles the behavior of sputtering yields observed in bulk channeling experiments along a low index direction of the crystal. ${ }^{33}$ The channeling direction, in this case, is parallel to the surface and thus equals $\vartheta_{\text {chan }}=90^{\circ}$ to the surface normal. Figure 8 allows us to determine the critical angle for surface channeling as $\vartheta_{\text {crit }}=73^{\circ}$. For bulk channeling, sputter yields $Y^{\text {chan }}\left(\vartheta_{\text {chan }}-\vartheta\right)$ have been described as the product of the corresponding amorphous yield $Y^{\text {amorph }}$ and the nonchanneling fraction $\chi\left(\vartheta_{\text {chan }}-\vartheta\right),{ }^{33-35}$

$$
Y^{\text {chan }}\left(\vartheta_{\text {chan }}-\vartheta\right)=f Y^{\text {amorph }} \chi\left(\vartheta_{\text {chan }}-\vartheta\right) \text {. }
$$

Here, $f$ is a fitting parameter of the order of unity (typically somewhat larger than 1), which accounts for the fact that for 


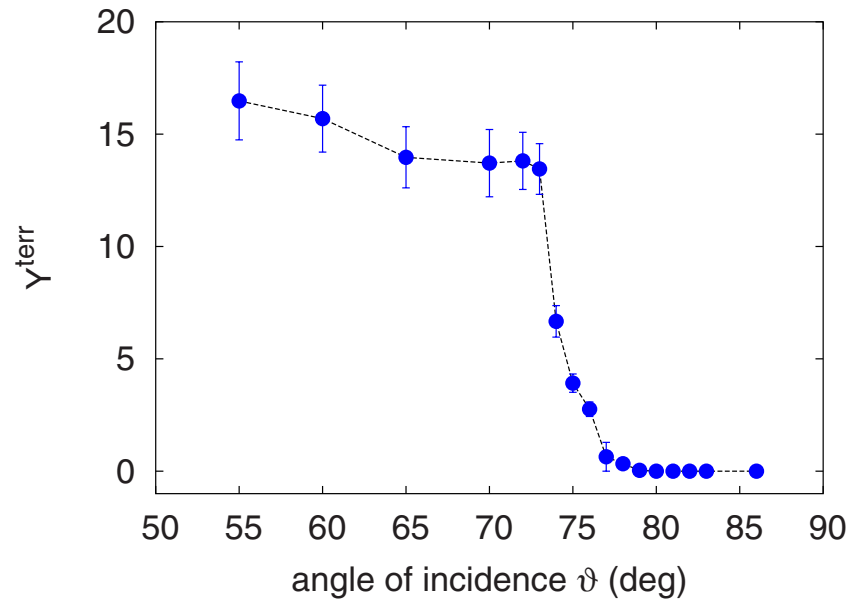

FIG. 8. (Color online) Terrace sputter yield $Y^{\text {terr }}$ for $5 \mathrm{keV} \mathrm{Ar}^{+}$ incident on $\mathrm{Pt}(111)$ at $0 \mathrm{~K}$ along the [1-12] azimuth for a wide range of polar angles $\vartheta$.

a nonchanneling fraction of 1 , single crystal yields are somewhat larger than $Y^{\text {amorph }}$. The nonchanneling fraction $\chi$ has a minimum for ions incident precisely along the channeling direction (i.e., for $\left.\vartheta=\vartheta_{\text {chan }}\right)$. It increases up to $\chi\left(\vartheta_{\text {chan }}\right.$ $\left.-\vartheta_{\text {crit }}\right)=1$ when channeling ceases. In the present case of surface channeling $\chi(0)=0$, i.e., for extreme grazing incidence, all ions are reflected from the surface without sputtering. In this view, the weakly angle dependent $Y^{\text {terr }}$ for $\vartheta$ $\leq 73^{\circ}$ is a good estimate for $f Y^{\text {amorph }}$.

The presence of terrace vacancy islands in Fig. 2, which is observed in experiments performed at $\vartheta=86^{\circ}$, appears to contradict the absence of sputtering and adatom production for $\vartheta \geq 84^{\circ}$ found in the simulation. However, the density of vacancy clusters on the terrace (away from preexisting steps) after a fluence of 0.25 MLE in Fig. 2 is only 9.0 $\times 10^{-6} \mathrm{~A}^{-2}$. Dividing the cluster density by the number of ions incident on the terrace yields a probability of $5 \times 10^{-4}$ for the initiation of vacancy cluster formation through terrace impacts. Assuming a vacancy cluster to result from a single adatom production event gives a lower bound estimate $Y^{\text {terr,ad }} \approx 5 \times 10^{-4}$. An upper bound estimate results from dividing the terrace area covered by vacancy islands of $0.2 \%$ by the ion fluence resulting in $Y^{\text {terr,ad }} \approx 5 \times 10^{-3}$. Both numbers are consistent with the limited statistics of the MD simulations. Moreover, as pointed out above, a very small concentration of adsorbed molecules from the background gas might also be of relevance in initiating vacancy island formation on terraces.

Figure 9 summarizes our simulation results for sputtering through $5 \mathrm{keV} \mathrm{Ar}^{+}$on a stepped $\mathrm{Pt}(111)$ surface. As the amounts of sputtering and adatom production are roughly proportional to each other, with the ratio of sputtered atoms to adatoms being $\cong 3-4,{ }^{16,23}$ we limit our discussion to the sputter yields here. We first concentrate our discussion on the $0 \mathrm{~K}$ data. The expectations for the zones of influence from the geometrical model for the $0 \mathrm{~K}$ yield are indicated by dashed lines in Fig. 9. Along the [112] azimuth, the geometrical zone of influence agrees rather well with an accuracy of about one lattice spacing with the $\xi$ range of signifi-

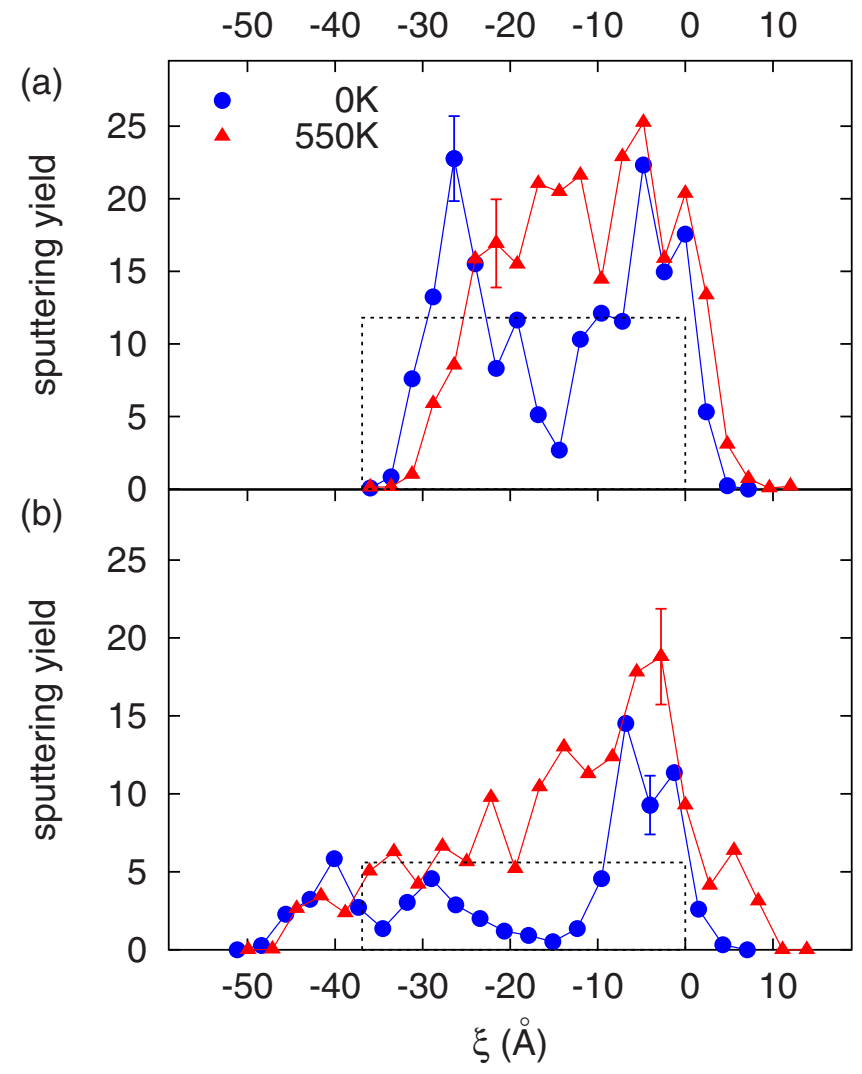

FIG. 9. (Color online) Sputtering yields of $5 \mathrm{keV} \mathrm{Ar}^{+}$on $\mathrm{Pt}$ (111) for $\vartheta=83^{\circ}$ as a function of the distance $\xi$ to the step edge. (a)

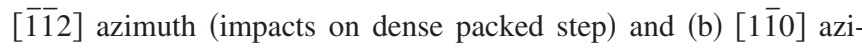
muth (impacts on kinked step). The dashed lines in (a) and (b) represent the estimate of $Y^{\text {step }}$ at $0 \mathrm{~K}$ according to the geometrical model and as obtained from the simulation results via Eq. (7).

cant sputtering according to the MD simulations. In contrast, along the $[1 \overline{1} 0]$ azimuth, the $\xi$ range of significant sputtering according to the MD simulations extends much further in front of the step than expected from the geometrical model. To obtain a clue on this MD result, in Figs. 10(a) and 10(b), the channels in a Pt crystal along the [112] azimuth and the

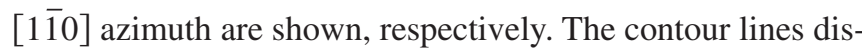
played indicate the turning points in the motion of a $5 \mathrm{keV}$ $\mathrm{Ar}^{+}$ion with $83^{\circ}$ incidence angle (perpendicular energy $E^{\perp}$ $=74 \mathrm{eV}$ ), i.e., at these positions, the ion is reflected from the channel walls. ${ }^{36}$ For ions in front of the step edge and outside the crystal, only the lower (red) contour lines are relevant. We note that the corrugation of the potential perpendicular to the plane of drawing is small; it amounts to only 0.053 (0.013) $\AA$ for the $[1 \overline{1} 0]([\overline{1} \overline{1} 2])$ azimuth. The energy of 74 $\mathrm{eV}$ corresponds to $E^{\perp}$ of the $5 \mathrm{keV} \mathrm{Ar}{ }^{+}$ions impinging at $\vartheta=83^{\circ}$. The corrugation of the contour lines amounts to

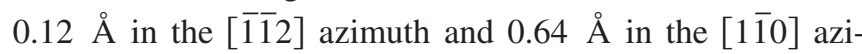
muth. Thus, ions impinging along the [ $\overline{1} \overline{1} 2]$ azimuth are guided by an almost planar potential in front of the step edge, whereas ions impinging along the $[1 \overline{1} 0]$ azimuth experience a corrugated potential. As a consequence, ions impinging on the ridges of the structure are reflected early, 

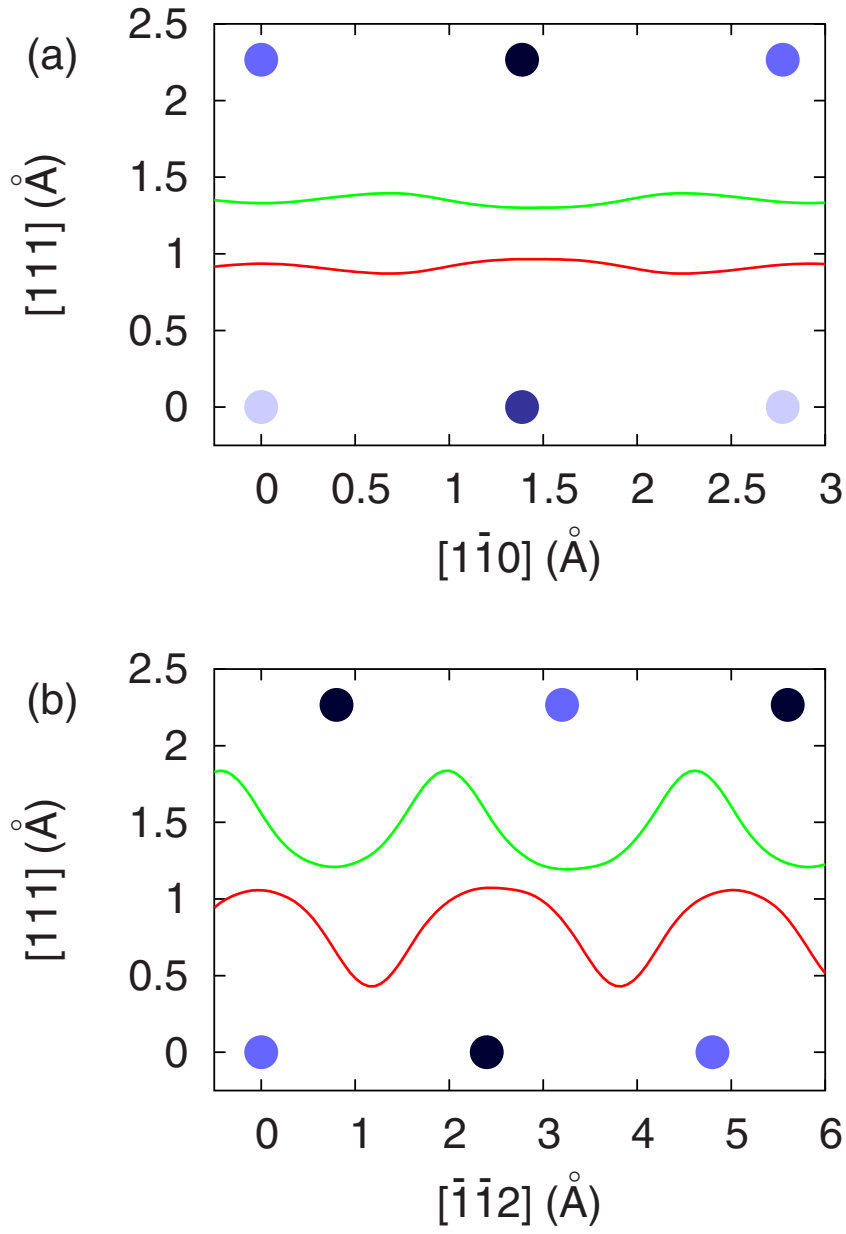

FIG. 10. (Color online) Representation of the contour lines at $E^{\perp}=74 \mathrm{eV}$ in the channels of a Pt crystal for (a) the [11-12] azimuth and (b) the [1힐 azimuth. $E^{\perp}$ is the perpendicular energy of $5 \mathrm{keV}$ $\mathrm{Ar}^{+}$with $\vartheta=83^{\circ}$. The view is along the projection of the ion direction onto the surface plane, i.e., the ions are moving into the plane of paper. The circles show the Pt atom positions and the different shadings indicate that the atoms have different positions in the direction normal to the paper plane.

while the ion trajectories become extended when they impinge into a valley. The surface corrugation in front of a kinked step therefore causes strongly dispersed ion trajectories and the $\xi$ range of significant sputtering along the [1 $\overline{1} 0]$ azimuth correspond less well with the zone of influence derived from the geometrical model. In agreement with this discussion, the total extent of the zone of influence-defined as the zone where ion impact leads to nonzero sputter yield-amounts to $43 \AA$ for ions along the [1-̄12] azimuth (impacting a $\{111\}$-microfacetted step) and to $58 \AA$ along the [1ํㅣㄹ azimuth (impacting a fully kinked step).

Figure 9 displays, for both azimuthal directions, a clear dependence of the sputtering yield on $\xi$, contrary to the expectation (i) from the geometric model (compare Sec II). The largest yields are obtained for ions directly impinging on step atoms with a small impact parameter and $\xi$ in the range of 0 to $-10 \AA$. Minima in the sputtering yield are observed for $\xi$ in the range of -10 to $-20 \AA$. We attribute these minima to subsurface channeling. Ions in this $\xi$ range enter the crystal with high probability at the step edge between the lower terrace and the upper terrace layer. At $0 \mathrm{~K}$, these ions are guided for long distances between the potentials of the two layers while losing energy through electronic excitations (not included in our simulations) and through damage production at the surface. Eventually, these ions dechannel through a large angle scattering event. At the point of dechanneling, typically, sputtering takes place and vacancy as well as adatom clusters result. Usually, the dechanneled ion leaves the crystal with a significant amount of kinetic energy which is lost for sputtering. The channeling dip at about $\xi=-15 \AA$ is considerably more pronounced along the $[1 \overline{1} 0]$ azimuth [Fig. 9(b)] compared to the [112] azimuth [Fig. 9(a)]. This difference may again be traced back to the differences in the $74 \mathrm{eV}$ contour lines shown in Fig. 10. Ions traveling along the [112] direction are guided in between planar potentials of the first and the second layers with only minor perturbations originating from the crystal atoms. Therefore, ions perform-

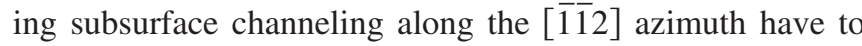
overcome the planar potential in order to dechannel. This transfers a significant amount of energy to the crystal, which results in sputtering at the dechanneling point. In contrast, along the $[1 \overline{1} 0]$ azimuth, the potential is not planar but strongly corrugated. Due to the strong corrugation, ions performing axial channeling along the [1히 ] azimuth are able to change their axial channel without significant energy loss. This implies not only that such ions may leave the crystal causing no or only little damage but also that these ions may even penetrate to deeper layers; both effects were seen in our simulations. ${ }^{37}$ They contribute to making the channeling dip of the $[1 \overline{1} 0]$ azimuth more pronounced.

The sputtering yield along the [112] azimuth has a bimodal structure with a second peak in the sputtering yield appearing for $\xi \cong-30$ to $-20 \AA$. This maximum shows up because in this $\xi$ range, the projectile atom hits the step after reflection (surface channeling) from the lower terrace [trajectory 1 in Fig. 1(a)]. This second maximum is considerably smaller for the [1피 azimuth as indirect hits lead to comparatively less sputtering. The rougher surface potential (Fig. 10) steers projectiles into axial channels on the lower terrace; these ions then have a decreased probability of hitting stepedge atoms, and hence, sputtering is reduced.

To compare the results of our MD simulations with the experimental $Y^{\text {step }}$, we integrate the $\xi$-dependent yields and divide by $x_{c}$ as derived from the geometrical model,

$$
Y^{\text {step }}=\frac{\int Y(\xi) d \xi}{x_{c}} .
$$

By using Eq. (7) at $0 \mathrm{~K}$, we obtain $Y^{\text {step }}=11.8$ for the [112] azimuth and $Y^{\mathrm{step}}=5.6$ for the $[1 \overline{1} 0]$ azimuth. This strong azimuthal dependence is mainly attributed to the fact that sputtering due to indirect hits is strongly reduced for the [1̄ㅣ] azimuth, cf. Fig. 9. 
TABLE I. Experimental data $(550 \mathrm{~K})$ and MD simulation results for $5 \mathrm{keV} \mathrm{Ar}^{+}$on $\mathrm{Pt}(111)$ for the [1]12] and the $[1 \overline{1} 0]$ azimuths.

\begin{tabular}{rccccc}
\hline \hline Angle $\vartheta$ & Beam direction & $Y_{\text {step }}$ & $\Delta_{Y_{\text {step }}}$ & MD (550 K) & MD (0 K) \\
\hline $83^{\circ}$ & {$[\overline{1} \overline{1} 2]$} & 7.7 & 0.9 & 15.8 & 11.8 \\
$82^{\circ}, 83^{\circ}$ & {$[1 \overline{1} 0]$} & 4.6 & 0.6 & 11.8 & 5.6 \\
$86^{\circ}$ & {$[\overline{1} \overline{1} 2]$} & 5.7 & 0.6 & NA & 10.7 \\
$86^{\circ}$ & {$[1 \overline{1} 0]$} & 4.5 & 0.9 & NA & 5.7 \\
\hline \hline
\end{tabular}

Taking temperature into account changes the picture (compare blue circles in Fig. 9). The most important effect of temperature is an increase in the yields, such that at $550 \mathrm{~K}$, $Y^{\text {step }}=15.8$ for the $[\overline{1} 12]$ azimuth and $Y^{\text {step }}=11.8$ for the

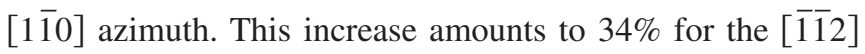
azimuth and to $112 \%$ for the [1피 azimuth. The increase results from step roughening and atomic vibrations, which significantly increase the probability of large angle scattering events. Such a dramatic influence of temperature on sputtering and damage formation has, to our knowledge, neither been experimentally observed nor been theoretically predicted for grazing incidence ion bombardment. This strong temperature dependence is at variance with the expectation (iii) of the geometric model that $Y^{\text {step }}$ is temperature independent (compare Sec II). Increases in the yields at $\xi=0$, i.e., when hitting the step edge directly, are primarily due to step roughening. Most pronouncedly, the channeling dip at $\xi \approx-15 \AA$ is largely filled for both azimuthal directions. Here, thermal roughening increases the probability for large scattering events while the ions are guided between the first two layers of the crystal. Subsurface channeling probabilities do not significantly decrease with increasing temperature ${ }^{5}$ however, the distance a particle channels is strongly reduced and more violent dechanneling events take place. ${ }^{38}$ We note that as the increase in yield with temperature for the [1] 0$]$ azimuth is stronger than that for the [1-12] one, the azimuthal anisotropy of the yield decreases toward higher temperature. We presume that for still higher temperatures, the surface will appear rather isotropic to the ion beam and all azimuthal dependence will disappear.

Finally, for $0 \mathrm{~K}$, we also investigated the angular dependence of $Y^{\text {step }}$ by using MD simulations. For the [1 10$]$ azimuth, the yield for $\vartheta=86^{\circ}$ is $Y^{\text {step }}=5.7$, which is within the limits of error identical to the one obtained for $\vartheta=83^{\circ}$. Similarly, the sputter yields for the [1-12] azimuth change only little. We note that we are investigating the angular dependence of the step-edge sputter yield over a wider range of incidence angles ${ }^{39}$; preliminary results suggest that for incidence angles $\vartheta \gtrsim 75^{\circ}, Y^{\text {step }}$ is rather constant, while it converges toward the terrace yield for steeper incidence angles. Thus, comparable to $Y^{\text {terr }}, Y^{\text {step }}$ also displays a behavior that is similar to bulk channeling with a minimum of the nonchanneling fraction for ions incident precisely along [112] $\left(\vartheta=90^{\circ}\right)$. Taking the yield for $\vartheta=80^{\circ}$ as an estimate for $f Y^{\text {amorph }}$, the nonchanneling fraction for incident ions parallel to the surface amounts to about $\chi(0) \approx 0.65$. We note that the bulk channeling concept must be considered with care here, as most of the channeling ions will also eventually dechannel and contribute to sputtering, though to a lesser extent.

\section{DISCUSSION}

The experimental and simulation results for $Y^{\text {step }}$ in both azimuthal directions are summarized in Table I. It is obvious from Table I that $Y^{\text {step }}$ depends on the azimuthal angle. In the range of $\vartheta=82^{\circ}-86^{\circ}$, experiments and simulations agree that in the $[1 \overline{1} 0]$ azimuth, $Y^{\text {step }}$ is significantly lower compared to the [1-12] azimuth by about $20 \%-30 \%$ at $550 \mathrm{~K}$ and even $50 \%$ for $0 \mathrm{~K}$ (simulations only). In the channeling picture, this difference is nothing but expected, since in an fcc metal, the $[1 \overline{1} 0]$ direction is the most transparent direction with the largest acceptance angle $\vartheta_{\text {chan }}-\vartheta_{\text {crit }}$ for channeling. The lower yields in the [1 $1 \overline{1} 0]$ azimuth are thus due to a larger channeling fraction of ions, which contribute less to sputtering. In fact, by using Onderdelinden's ${ }^{33,34}$ transparency concept, we can calculate the nonchanneling fraction of $5 \mathrm{keV}$ $\mathrm{Ar}^{+}$in $\mathrm{Pt}$ as $\chi(0)=0.3$ for the $[1 \overline{1} 0]$ azimuth and $\chi(0)=0.7$ for the $[\overline{1} 12]$ azimuth. ${ }^{40}$ The channeling ions contribute less to sputtering as they lose energy through less violent mechanisms (electronic stopping and nonsputtering surface damage production) and eventually leave the crystal again with a high probability and a significant amount of energy through dechanneling; this energy is lost for sputtering. Contrary to bulk sputtering, ions performing subsurface channeling are not lost entirely for sputtering. Therefore, the anisotropy of the nonchanneling fraction overestimates the anisotropy of the step-edge yields.

One of the key results of the MD simulations is the temperature dependence of $Y^{\text {step }}$. As visible from Table I, $Y^{\text {step }}$ drops by up to $50 \%$ due to lowering of the temperature from 550 to $0 \mathrm{~K}$. This decrease is caused by thermal vibrations, which reduce the channeling fraction of particles. A similar, though weaker, dependence of sputtering on temperature was also found for nongrazing incidence directions close to a channeling direction. ${ }^{33}$ The decrease, in this case, is essentially caused by a decrease in the penetration depth of the ions due to thermal vibrations of the crystal atoms. ${ }^{41}$ More energy is transferred closer to the surface, resulting in an increase in the sputtering yields. Future experimental and theoretical works are certainly necessary to fully exploit the mechanisms and the relevance of the temperature dependence of sputtering under grazing incidence conditions. 
According to Table I, $Y^{\text {step }}$ depends on the polar angle $\vartheta$ along the $[\overline{1} 12]$ azimuth but not or to a much lesser extent for the $[1 \overline{1} 0]$ azimuth. Using the channeling picture again, this fact may be understood from the azimuth dependence of the critical angle for channeling. Lindhard ${ }^{42}$ calculated the acceptance angle for channeling to be approximately

$$
\psi_{2}=\left(\frac{3 Z_{1} Z_{2} e^{2}}{4 \pi \varepsilon_{0}} \frac{a^{2}}{d^{3} E}\right)^{1 / 4},
$$

where $Z_{1}$ and $Z_{2}$ are the atomic number of projectile and target atom, respectively, $E$ is the projectile energy, and $d$ is the interatomic distance along the atomic string. $a$ $=0.8853 a_{0} /\left(Z_{1}^{2 / 3}+Z_{2}^{2 / 3}\right)^{1 / 2}$ denotes the Thomas-Fermi screening radius and $a_{0}$ is Bohr's radius. The acceptance angle $\psi_{2}=\vartheta_{\text {chan }}-\vartheta_{\text {crit }}$ for channeling of $5 \mathrm{keV} \mathrm{Ar}^{+}$in Pt amounts to $15^{\circ}$ for the $[1 \overline{1} 0]$ azimuth and about $10^{\circ}$ for the [1112] azimuth. The nonchanneling fraction $\chi\left(\vartheta_{\text {chan }}-\vartheta\right)$ has a minimum for $\vartheta=\vartheta_{\text {chan }}$ and varies close to this value only weakly before it increases steeply to reach 1 for $\vartheta=\vartheta_{\text {crit }}$. As the acceptance $\vartheta_{\text {chan }}-\vartheta_{\text {crit }}$ is $15^{\circ}$ for the [1 $\left.1 \overline{1} 0\right]$ azimuth, the nonchanneling fraction for the angles $\vartheta=82^{\circ}-86^{\circ}$ is still close to its minimum value. In contrast, $\vartheta_{\text {chan }}-\vartheta_{\text {crit }}$ amounts to

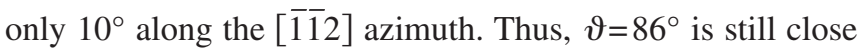
to $\chi(0)$, whereas the angle $\vartheta=83^{\circ}$ is already on the slope to $\chi\left(\vartheta_{\text {chan }}-\vartheta_{\text {crit }}\right)=1$. Consequently, $Y^{\text {step }}$ varies more in the range of $\vartheta=83^{\circ}-86^{\circ}$ for the [i $\left.1 \overline{1} 2\right]$ azimuth.

The trends of MD simulations and experiment are consistent with each other; however, the MD simulations tend to overestimate the experimental yields roughly by a factor of 2. Similar deviations between simulation and experiment are known from previous work. ${ }^{29,43-45}$ The origin of this quantitative discrepancy should mainly be attributed to an incomplete knowledge of the interatomic interaction potential of Pt. Interatomic potentials are usually fitted to the bulk properties of the material; this also applies to the Pt potential used here; they describe surface properties less reliably. In the present application, however, the properties of surfaces and surface defects play a major role. We also mention that electronic stopping was ignored in our simulation; this may affect, in particular, long-ranged channelled projectile trajectories and may lead to an overestimation of sputter yields. Two further issues may affect the quantitative reliability of a molecular-dynamics simulation: (i) our crystallite is too small to contain all projectile trajectories, even though we extended it as much as it appeared justifiable (computing times are of the order of a few weeks for each polar angle and azimuthal direction on a 100 node PC cluster in order to gather sufficient statistics). We estimate that around $37 \%$ of the ion trajectories for $83^{\circ}$ incidence in the [ $\left.\overline{1} \overline{1} 2\right]$ azimuth are not contained in our crystallite, i.e., the ion trajectories leave the crystallite via subsurface channeling along a side. Hence, the finite size of the simulation volume underestimates the sputter yields by (at most) about $37 \%$. The corresponding numbers for the $[1 \overline{1} 0]$ azimuth are not known to us. (ii) Each molecular-dynamics simulation neglects the quantum mechanical zero-point motion of atoms at low temperatures. Typically, above the Debye temperature (240 K for Pt), ther- mal vibrations may be treated using classical physics, which gives confidence to our $550 \mathrm{~K}$ results. However, our $0 \mathrm{~K}$ simulations treat the crystal in an idealized way as completely motionless; in the real low-temperature solid, the atoms would not be on ideal lattice positions; the resulting disorder would increase the sputter yield.

By comparing the present experimental results for the [1112] azimuth at $83^{\circ}$ with our previous experimental results, ${ }^{15}$ the agreement is satisfactory. The present value obtained at $550 \mathrm{~K}, Y^{\text {step }}=7.7 \pm 0.9$ agrees within the limits of error with the previous one of $Y^{\text {step }}=8.4 \pm 1.5$ obtained at $720 \mathrm{~K}$. The somewhat higher yield resulting from the higher temperature experiments could be due to the increase in $Y^{\text {step }}$ with temperature because of the amplitude increase in thermal vibrations.

Finally, our discussion also allows one to straightforwardly obtain an estimate of the erosion rate $\bar{Y}$ at grazing incidence. Under grazing incidence conditions, $Y^{\text {terr }}$ is of the order of zero. Therefore, $\rho_{\text {step }} x_{c}$, which is the product of step density $\rho_{\text {step }}$ and the width of the zone of influence in front of an illuminated step, gives the fraction of the surface area subject to ion sputtering. The product of this fraction of surface area subject to sputtering with the step edge yield $Y^{\text {step }}$ is the average erosion rate $\bar{Y}$ [Eq. (1)].

We propose that $Y^{\text {step }}$ itself may be well approximated by a TRIM calculation for $Y^{\operatorname{amorph}}(\vartheta)$ at the chosen angle of incidence. ${ }^{14}$ TRIM grossly fails to calculate the average yield $\bar{Y}(\vartheta)$ for grazing incidence, as it does not take into account surface channeling. Even under grazing incidence, TRIM calculates for every impact random collision cascades involving large angle scattering events. Such cascades are characteristic for step-edge impacts but not for surface impacts. Therefore, the source of failure, making TRIM calculations unsuitable to estimate $\bar{Y}$ at grazing incidence, makes them suitable to estimate $Y^{\text {step }}$ under these conditions. At grazing incidence, TRIM accounts, in a reasonable way, for energy loss through the primary ion or other high energy recoils scattered into the vacuum. However, the TRIM values derived in such a way for $Y^{\text {step }}$ suffer from two fundamental deficits, which fortunately cause errors of opposite sign. The amorphous yield calculated by TRIM generally underestimates the crystalline yield obtained for identical parameters in a nonchanneling direction..$^{29,33}$ As an example, the normal incidence yield of $5 \mathrm{keV} \mathrm{Ar}{ }^{+}$onto $\mathrm{Pt}(111)$ is calculated by TRIM to be 5.4, while the experimental result is 7.4. For the same reason, TRIM neglects subsurface channeling and, therefore, tends to overestimate the experimental yield. As both errors are of opposite sign and similar magnitude, they tend to cancel. For example, TRIM yields for $Y^{\text {amorph }}\left(83^{\circ}\right)=6.5$, which reasonably compares well with the values of 7.7 and 4.6 (see Table I) for the [ $\overline{1} \overline{1} 2]$ and $[1 \overline{1} 0]$ azimuth, respectively. Our simple estimate can be expected to predict the grazing incidence erosion rate in a wide parameter range correctly to within a factor of 2. Better estimates may be based on Eq. (6). However, these estimates also have to be treated with care as subsurface channeling is different from bulk channeling. Channeling particles are not entirely lost for sputtering and the extent to which these particles contribute to sputtering depends strongly on temperature. 


\section{CONCLUSIONS}

With the onset of surface channeling for grazing incidence, ion sputtering from flat surface regions of crystals ceases and the average erosion rate is exclusively determined by sputtering of step edges illuminated by the ion beam. The sputtering of these steps results not only from direct impacts but also from ions reflected by surface channeling on the lower terrace near the step. Here, we introduced and applied two new experimental methods to obtain the step-edge yield $Y^{\text {step }}$ under grazing incidence conditions. The results of these methods are consistent with each other and also agree with our previous measurements. ${ }^{15}$ Step-edge yields display a clear dependence on the azimuthal angle. Our molecular dynamics simulations evidence that this dependence is due to the azimuthal dependence of surface and subsurface channel- ing. They also display a strong dependence of $Y^{\text {step }}$ on temperature, which is primarily caused by the influence of vibrations on channeling. A simple procedure to estimate the average erosion rate under grazing incidence is given, which needs, as input, only the step concentration and the sputtering yield as calculated by TRIM for the chosen angle of incidence.

\section{ACKNOWLEDGMENTS}

We acknowledge useful discussions with Oliver Ricken and Carsten Busse. This work was supported by the Deutsche Forschungsgemeinschaft via the project "Atomic scale surface damage by ion bombardment at grazing incidence" and by TPSDP Ministry of National Education, Republic of Indonesia. *redinger@ph2.uni-koeln.de

${ }^{1} \mathrm{~S}$. van Dijken, D. de Bruin, and B. Poelsema, Phys. Rev. Lett. 86, 4608 (2001).

${ }^{2}$ S. Rusponi, C. Boragno, and U. Valbusa, Phys. Rev. Lett. 78, 2795 (1997).

${ }^{3}$ B. Ziberi, F. Frost, T. Höche, and B. Rauschenbach, Vacuum 81, 155 (2006).

${ }^{4}$ H. Hansen, A. Redinger, S. Meßlinger, G. Stoian, Y. Rosandi, H. M. Urbassek, U. Linke, and T. Michely, Phys. Rev. B 73, 235414 (2006).

${ }^{5}$ A. Redinger, H. Hansen, U. Linke, Y. Rosandi, H. M. Urbassek, and T. Michely, Phys. Rev. Lett. 96, 106103 (2006).

${ }^{6}$ W. L. Chan and E. Chason, J. Appl. Phys. 101, 121301 (2007).

${ }^{7}$ P. Chaudhari, J. Lacey, J. Doyle, E. Galligan, S. C. A. Lien, A. Callegari, G. Hougham, N. D. Lang, P. S. Andry, R. John, K. H. Yang, M. H. Lu, C. Cai, J. Speidell, S. Purushothaman, J. Ritsko, M. Samant, J. Stohr, Y. Nakagawa, Y. Katoh, Y. Saitoh, K. Sakai, H. Satoh, S. Odahara, H. Nakano, J. Nakagaki, and Y. Shiota, Nature (London) 411, 56 (2001).

${ }^{8}$ R. Moroni, D. Sekiba, F. Buatier de Mongeot, G. Gonella, C. Boragno, L. Mattera, and U. Valbusa, Phys. Rev. Lett. 91, 167207 (2003)

${ }^{9}$ L. Vattuone, U. Burghaus, L. Savio, M. Rocca, G. Costantini, F. Buatier de Mongeot, C. Boragno, S. Rusponi, and U. Valbusa, J. Chem. Phys. 115, 3346 (2001).

${ }^{10}$ B. Enders, R. Emmerich, and W. Ensinger, Surf. Coat. Technol. 66, 478 (1994).

${ }^{11}$ K. C. Ruthe and S. A. Barnett, Surf. Sci. 538, L460 (2003).

${ }^{12}$ P. Sigmund, Phys. Rev. 184, 383 (1969).

${ }^{13}$ Y. Yamamura and H. Tawara, At. Data Nucl. Data Tables 62, 149 (1996).

${ }^{14} \mathrm{http}: / /$ www.srim.org

${ }^{15}$ H. Hansen, C. Polop, T. Michely, A. Friedrich, and H. M. Urbassek, Phys. Rev. Lett. 92, 246106 (2004).

${ }^{16}$ A. Friedrich and H. M. Urbassek, Surf. Sci. 547, 315 (2003).

${ }^{17}$ H. Winter, Phys. Rep. 367, 387 (2002).

${ }^{18}$ A. A. Dzhurakhalov, Microelectron. Eng. 69, 570 (2003).

${ }^{19}$ M. Bott, T. Michely, and G. Comsa, Rev. Sci. Instrum. 66, 4135 (1995).
${ }^{20}$ T. Michely and G. Comsa, Phys. Rev. B 44, 8411 (1991).

${ }^{21}$ T. Michely and C. Teichert, Phys. Rev. B 50, 11156 (1994).

${ }^{22}$ C. Busse, C. Engin, H. Hansen, U. Linke, T. Michely, and H. M. Urbassek, Surf. Sci. 488, 346 (2001).

${ }^{23}$ H. Gades and H. M. Urbassek, Phys. Rev. B 50, 11167 (1994).

${ }^{24}$ J. F. Ziegler, J. P. Biersack, and U. Littmark, The Stopping and Range of Ions in Solids (Pergamon, New York, 1985).

${ }^{25}$ Y. Rosandi and H. M. Urbassek, Surf. Sci. 600, 1260 (2006).

${ }^{26}$ M. Kalff, G. Comsa, and T. Michely, Surf. Sci. 486, 103 (2001).

${ }^{27}$ Koen Schoots, Ph.D. thesis, University Leiden, 2007.

${ }^{28}$ For a given fluence the average distance traveled by a preexisting step in ion beam direction does not or only marginally depend on the step orientation, as long angle between step normal and the projection of the ion beam on the surface is less than $40^{\circ}$.

${ }^{29}$ T. Michely and C. Teichert, Phys. Rev. B 50, 11156 (1994).

${ }^{30}$ To a certain extent the larger vacancy island density island is also caused by the flux larger by a factor of 2 for $\vartheta=82-83^{\circ}$ in comparison to that of $\vartheta=86^{\circ}$.

${ }^{31}$ C. Busse, H. Hansen, U. Linke, and T. Michely, Phys. Rev. Lett. 85, 326 (2000).

${ }^{32}$ A. Petersen, C. Busse, C. Polop, U. Linke, and T. Michely, Phys. Rev. B 68, 245410 (2003).

${ }^{33}$ H. E. Roosendaal, in Sputtering by Particle Bombardment, edited by R. Behrisch (Springer, Berlin 1981), Vol. 1, p. 219.

${ }^{34}$ D. Onderdelinden, Appl. Phys. Lett. 8, 189 (1966).

${ }^{35}$ W. Pamler, K. Wangemann, S. Kampermann, and W. Hosler, Nucl. Instrum. Methods Phys. Res. B 51, 34 (1990).

${ }^{36} \mathrm{We}$ feel that this presentation of the channel has advantages over the usual plots of equipotential lines of the continuum potential, since our presentation includes the effect of atom shadowing, which is important at small perpendicular energies and for heavy projectiles.

${ }^{37} \mathrm{http}: / / w w w . p h y s i k . u n i-k l . d e /$ urbassek

${ }^{38}$ Y. Rosandi and H. M. Urbassek, Nucl. Instrum. Methods Phys. Res. B 256, 373 (2007).

${ }^{39}$ Y. Rosandi, H. M. Urbassek, A. Redinger, and T. Michely (unpublished).

${ }^{40}$ These values are obtained using $\chi(0)=\pi n d^{3} \psi_{2}^{2}$, where the acceptance angle $\psi_{2}$ is given by Eq. (8), $n$ is the atomic number 
density of the crystal, and $d$ is the interatomic distance along the atomic string (Refs. 33 and 34).

${ }^{41}$ J. J. PH. Elich, H. E. Roosendaal, and D. Onderdelinden, Radiat. Eff. 10, 175 (1971).

${ }^{42}$ J. Lindhard, Mat. Fys. Medd. K. Dan. Vidensk. Selsk. 34, 14 (1965).
${ }^{43}$ H. Gades and H. M. Urbassek, Phys. Rev. B 50, 11167 (1994).

${ }^{44}$ M. Morgenstern, T. Michely, and G. Comsa, Philos. Mag. A 79, 775 (1999).

${ }^{45}$ M. Ghaly, K. Nordlund, and R. S. Averback, Philos. Mag. A 79, 795 (1999). 